Article

\title{
Asymptotic Stabilization of Delayed Linear Fractional-Order Systems Subject to State and Control Constraints
}

\author{
Xindong Si $(\mathbb{D}$, Zhen Wang * $\mathbb{D}$, Zhibao Song and Ziye Zhang \\ College of Mathematics and Systems Science, Shandong University of Science and Technology, \\ Qingdao 266590, China; sixindongsk@163.com (X.S.); szb879381@163.com (Z.S.); zhangzy02@126.com (Z.Z.) \\ * Correspondence: wangzhen_sd@126.com
}

Citation: Si, X.; Wang, Z.; Song, Z.; Zhang, Z. Asymptotic Stabilization of Delayed Linear Fractional-Order Systems Subject to State and Control Constraints. Fractal Fract. 2022, 6, 67. https://doi.org/10.3390/

fractalfract6020067

Academic Editors:António M. Lopes and Liping Chen

Received: 24 December 2021

Accepted: 25 January 2022

Published: 27 January 2022

Publisher's Note: MDPI stays neutral with regard to jurisdictional claims in published maps and institutional affiliations.

Copyright: (C) 2022 by the authors. Licensee MDPI, Basel, Switzerland. This article is an open access article distributed under the terms and conditions of the Creative Commons Attribution (CC BY) license (https:// creativecommons.org/licenses/by/ $4.0 /)$.

\begin{abstract}
Studies have shown that fractional calculus can describe and characterize a practical system satisfactorily. Therefore, the stabilization of fractional-order systems is of great significance. The asymptotic stabilization problem of delayed linear fractional-order systems (DLFS) subject to state and control constraints is studied in this article. Firstly, the existence conditions for feedback controllers of DLFS subject to both state and control constraints are given. Furthermore, a sufficient condition for invariance of polyhedron set is established by using invariant set theory. A new Lyapunov function is constructed on the basis of the constraints, and some sufficient conditions for the asymptotic stability of DLFS are obtained. Then, the feedback controller and the corresponding solution algorithms are given to ensure the asymptotic stability under state and control input constraints. The proposed solution algorithm transforms the asymptotic stabilization problem into a linear/nonlinear programming (LP/NP) problem which is easy to solve from the perspective of computation. Finally, three numerical examples are offered to illustrate the effectiveness of the proposed method.
\end{abstract}

Keywords: delayed linear fractional-order systems; feedback controller; positive invariant set; asymptotic stabilization

\section{Introduction}

Fractional calculus almost appeared at the same time as classic calculus, but it has not been paid more attention to due to its lack of application background and difficult calculation. Fractional calculus has experienced rapid development during the last few decades both in mathematics and applied sciences. It has been recognized as an excellent tool to describe modern complex dynamics [1,2]. From this perspective, some models governing physical phenomena have been reformulated in light of fractional calculus to better reflect their non-local, frequency- and history-dependent properties. With the rapid development of computer technology, fractional calculus is widely used in many fields, such as image processing [3], fluid mechanics [4], and environmental science [5]. Time delay often occurs in different practical systems, and the delayed fractional-order system can better describe these phenomena [6-8].

However, time delay will lead to system performance degradation, poor stability and even failure to work $[9,10]$. In fact, many scholars have extensively studied the stability of delayed fractional-order systems. For instance, the finite-time stability was discussed in [11-14]. Using the Laplace transform method, the globally asymptotic stability was studied in [15]. The Mittag-Leffler stability was discussed in [16,17]. The asymptotic stability was investigated using the frequency domain method [18], integral inequality method [19], linear matrix inequality (LMI) method [20], and Lyapunov function method [21,22].

On the other hand, for security reasons or physical constraints, the control input, the state, and/or output variables must be bounded in practice. That is to say, the hard constraints of these variables should be considered. At this time, how to design a feedback controller to ensure the stability of the system under constraints is an interesting topic 
in system theory and synthesis. For constrained integer-order systems, state feedback controllers were designed to study the asymptotic stability of linear discrete-time systems and linear continuous-time systems under constraints in [23,24]. Based on the LMI method, the optimization problem of state feedback controllers for linear discrete-time systems and continuous-time systems was further analyzed in $[25,26]$. A more effective method to solve the state feedback controller was developed based on the invariance of polyhedron set in $[27,28]$. As for unconstrained fractional-order systems, in [29], the global stabilization of fractional-order neural network was investigated by using the positive-system-based method. In [30], the stabilization of fractional-order T-S fuzzy systems was discussed by using LMI method. In [31], based on Lyapunov functions, a state feedback controller was designed to study the stabilization of fractional-order nonlinear systems. However, there are few studies concerning the stabilization problems of fractional-order system with constraints, except [32]. In [32], the stabilization problem of fractional-order linear systems with control input constraints was addressed by using the invariance of polyhedron set, but state constraints are not considered there.

Based on the above discussions, it is not only necessary but also more challenging to study the stabilization problem of DLFS under constraints. In this article, the stabilization problem of DLFS with state and control constraints is studied. Our main contributions include: (1) The sufficient conditions that ensure the state constraint set and/or the control constraint set are positive invariant sets (PIS) are established by using the invariant set theory; (2) A new Lyapunov function is constructed on the basis of the constraints, and the asymptotic stability conditions for DLFS are obtained; and (3) A feedback controller and its solution algorithm are proposed to make DLFS under the state and control input constraints asymptotically stable.

The article is organized as follows. Some preliminaries and the problem formulation are given in Section 2. In Section 3, existence conditions for the PIS and the feedback controller are developed. A feedback controller and its solution algorithm are proposed in Section 4 to ensure the DLFS under state and control constraints asymptotically stable. To illustrative the effectiveness of the proposed method, three examples are presented in Section 5. Section 6 contains some conclusions.

Notations: In this article, $\mathbb{R}_{+}$denotes the set of positive real numbers, $\mathbb{R}^{n}$ denotes $n$ dimensional real vector space, $\mathbb{R}^{n \times n}$ denotes $n \times n$ real matrices. $z$ stands for a complex number and $\operatorname{Re}(z)$ stands for the real part of $z . \rho_{i}$ represents the $i$ th element of vector $\rho$, and $Q_{i}$ represents the $i$ th row of matrix $Q, Q_{i j}$ represents the $i$ th row and $j$ th column element of matrix $Q$. For $\rho \in \mathbb{R}^{n}, \rho \geqslant 0(\rho>0)$ means $\rho_{i} \geqslant 0\left(\rho_{i}>0\right)$. For $a \in \mathbb{R}^{n}, b \in \mathbb{R}^{n}, a \geqslant b(a>b)$ means $a_{i} \geqslant b_{i}\left(a_{i}>b_{i}\right)$. $A>0$ indicates that each entry of the matrix $A$ is nonnegative.

\section{Preliminaries and Problem Formulation}

\subsection{Preliminaries}

Consider the DLFS:

$$
\left\{\begin{array}{l}
{ }_{0}^{C} D_{t}^{\alpha} x(t)=A x(t)+A_{0} x(t-\tau)+B u(t), \quad t>0, \\
x(t)=x_{0}, \quad-\tau \leqslant t \leqslant 0,
\end{array}\right.
$$

where $0<\alpha \leqslant 1, \tau=$ constant is the time delay, $A \in \mathbb{R}^{n \times n}$ and $A_{0} \in \mathbb{R}^{n \times n}$ are the system matrices, $B \in \mathbb{R}^{n \times m}$ is the input matrix, $x(t) \in \mathbb{R}^{n}$ is the state, and $u(t) \in \mathbb{R}^{m}$ is the control input.

Definition 1 ([12]). The Caputo fractional derivative of $x(t)$ is defined as

$$
{ }_{0}^{C} D_{t}^{\alpha} x(t)=\frac{1}{\Gamma(1-\alpha)} \int_{0}^{t}(t-s)^{-\alpha} x^{\prime}(s) d s,
$$

where the order $0<\alpha \leqslant 1$ and $\Gamma(z)=\int_{0}^{\infty} e^{-t} t^{z-1} d t, \operatorname{Re}(z) \in \mathbb{R}_{+}$. 
Definition 2 ([33]). The fractional integral of $x(t)$ is defined as

$$
{ }_{0} I_{t}^{\alpha} x(t)=\frac{1}{\Gamma(\alpha)} \int_{0}^{t}(t-s)^{\alpha-1} x(s) d s,
$$

where the order $\alpha \in \mathbb{R}_{+}$.

By the above Definition, we obtain the solution of system (1) as

$$
\begin{aligned}
x(t) & =x_{0}+{ }_{0} I_{t}^{\alpha}\left({ }_{0}^{C} D_{t}^{\alpha} x(t)\right) \\
& =x_{0}+\frac{1}{\Gamma(\alpha)} \int_{0}^{t}(t-s)^{\alpha-1}\left[A x(s)+A_{0} x(s-\tau)+B u(s)\right] d s
\end{aligned}
$$

Definition $3([34])$. A continuous function $\beta(x):[0,+\infty) \longrightarrow[0,+\infty)$ is said to be a class- $\kappa$ function if the function $\beta(x)$ is strictly increasing and $\beta(0)=0$.

Definition 4 ([29]). A nonempty set $P$ is called the positive invariant sets (PIS) if and only if

$$
x_{0} \in P \text { implies } x(t) \in P, \text { for } t>0 \text {, }
$$

where $x(t)$ is the trajectory starting with the initial value $x_{0}$.

Lemma 1 ([34]). Assume that there exist class- $\kappa$ functions $\beta_{i}, i=1,2,3$ and a continuously differentiable function $V(x(t))$ such that:

$$
\beta_{1}(\|x(t)\|) \leqslant V(x(t)) \leqslant \beta_{2}(\|x(t)\|),
$$

and

$$
{ }_{0}^{C} D_{t}^{\alpha} V(x(t)) \leqslant-\beta_{3}(\|x(t)\|),
$$

where the order $0<\alpha<1$, then system (1) is guaranteed to be stable. If $\beta_{3}(s)>0$ for $s>0$, then system (1) is guaranteed to be asymptotically stable.

Lemma 2 ([35]). The polyhedron set $P(Q, \rho)=\left\{x(t) \in \mathbb{R}^{n}: Q x(t) \leqslant \rho\right\}, Q \in \mathbb{R}^{q \times n}$, $\rho \in \mathbb{R}^{q}, \rho>0$, and $P(K, \omega)=\left\{x(t) \in \mathbb{R}^{n}: K x(t) \leqslant \omega\right\}, K \in \mathbb{R}^{m \times n}, \omega \in \mathbb{R}^{m}, \omega>0$ have the relation

$$
P(Q, \rho) \subseteq P(K, \omega)
$$

if and only if there exists $L \in \mathbb{R}^{m \times q}, L>0$ such that

$$
\left\{\begin{array}{l}
L Q=K \\
L \rho \leqslant \omega .
\end{array}\right.
$$

\subsection{Problem Formulation}

In this article, the following assumptions are needed.

Assumption 1. The state variables are constrained by the polyhedron set

$$
P(Q, \rho)=\left\{x(t) \in \mathbb{R}^{n}: Q x(t) \leqslant \rho\right\},
$$

where $Q \in \mathbb{R}^{q \times n}, q>n$, and $\rho \in \mathbb{R}^{q}, \rho>0$, and the polyhedron set $P(Q, \rho)$ is closed and nonempty.

Assumption 2. The control input $u(t)$ satisfies the following constraints

$$
-w_{1} \leqslant u(t) \leqslant w_{2}
$$

where $w_{1}>0$ and $w_{2}>0$ are real vectors. 
The asymptotic stabilization problem of DLFS (1) is to find a state feedback controller $u(t)=K x(t)$ that makes all trajectories starting with the initial value $x_{0}$ asymptotically stable and also satisfy the state constraints (5) and the control constraints (6).

If there exists $u(t)=K x(t)$ for system (1), then, by linear constraints (6), we obtain

$$
P\left(K,-w_{1}, w_{2}\right)=\left\{x(t) \in \mathbb{R}^{n}:-w_{1} \leqslant K x(t) \leqslant w_{2}\right\} .
$$

Let $\bar{A}=A+B K$; clearly, $u(t)=K x(t)$ is the solution of the asymptotic stabilization problem of system (1) if and only if the closed-loop system

$$
\left\{\begin{array}{l}
{ }_{0}^{C} D_{t}^{\alpha} x(t)=\bar{A} x(t)+A_{0} x(t-\tau), \quad t>0, \\
x(t)=x_{0}, \quad-\tau \leqslant t \leqslant 0
\end{array}\right.
$$

is asymptotically stable, and the trajectory $x(t)$ starting with $x_{0}$ satisfy $x(t) \in P(Q, \rho)$ and $x(t) \in P\left(K,-w_{1}, w_{2}\right)$ for any $t \geqslant 0$.

\section{Main Results}

3.1. Existence Conditions for the Feedback Controller with the Constraints

Theorem 1. The controller $u(t)=K x(t), K \in \mathbb{R}^{m \times n}$ is the solution of the asymptotic stabilization problem of system (1) if

(i) There exists a PIS denoted by $M \in \mathbb{R}^{n}$ for the system (8) satisfies $M \subseteq P(Q, \rho)$ and $M \subseteq P\left(K,-w_{1}, w_{2}\right)$;

(ii) There exists a Lyapunov function that makes the system (8) asymptotically stable.

Proof. Condition (i) ensures that there exists a PIS

$$
M=\left\{x_{0} \in \mathbb{R}^{n}: \forall x_{0} \in M, x(t) \triangleq x\left(t ; x_{0}\right) \in M\right\}
$$

such that $M \subseteq P(Q, \rho)$ and $M \subseteq P\left(K,-w_{1}, w_{2}\right)$, where $x\left(t ; x_{0}\right)$ is the trajectory starting with $x_{0}$. Then we can obtain $Q x(t) \leqslant \rho,-w_{1} \leq K x(t) \leqslant w_{2}$. Furthermore, the condition (ii) ensures that system (8) is asymptotically stable. Hence, the controller $u(t)=K x(t)$ is the solution of the asymptotic stabilization problem of system (1).

When considering both the state and control constraints, let $P(Q, \rho)$ be the PIS of system (8), that is, $P(Q, \rho)$ is equal to $M$, then we obtain:

Corollary 1. The controller $u(t)=K x(t), K \in \mathbb{R}^{m \times n}$ is the solution of the asymptotic stabilization problem of system (1) if

(i) $P(Q, \rho)$ is a PIS of the system (8) and $P(Q, \rho) \subseteq P\left(K,-w_{1}, w_{2}\right)$;

(ii) There exists a Lyapunov function that makes the system (8) asymptotically stable.

Moreover, if considering only the control constraints, let $P\left(K,-w_{1}, w_{2}\right)$ be the PIS of system (8), that is, $P\left(K,-w_{1}, w_{2}\right)$ is equal to $M$, we obtain:

Corollary 2. The controller $u(t)=K x(t), K \in \mathbb{R}^{m \times n}$ is the solution of the asymptotic stabilization problem of system (1) if

(i) $P\left(K,-w_{1}, w_{2}\right)$ is a PIS of the system (8);

(ii) There exists a Lyapunov function that makes the system (8) asymptotically stable.

\subsection{PIS and Stability Conditions for System (8)}

Theorem 2. If there exists a matrix $K \in \mathbb{R}^{m \times n}$, real matrices $F, F_{0} \in \mathbb{R}^{q \times q}$ and a scalar $\varepsilon>0$ such that

$$
\left\{\begin{array}{c}
Q(A+B K)=F Q \\
Q A_{0}=F_{0} Q \\
\left(F+F_{0}\right) \rho \leqslant-\varepsilon \rho
\end{array}\right.
$$


then the system (8) is asymptotically stable and the polyhedron set $P(Q, \rho)$ is a PIS.

Proof. Choose the Lyapunov function of the form

$$
V(x(t))=\max \left\{\max \left(\frac{Q_{1} x(t)}{\rho_{1}}, 0\right), \cdots, \max \left(\frac{Q_{q} x(t)}{\rho_{q}}, 0\right)\right\} .
$$

If, for $1 \leqslant i \leqslant q$, it satisfies $\frac{Q_{i} x(t)}{\rho_{i}}<0$, we obtain $V(x(t))=0$. However, due to the fact that $Q_{i} x(t)$ cannot always be negative, this case does not exist, so $V(x(t))>0$. Hence, there must exist an $i$, such that $0<Q_{i} x(t) \leqslant \rho_{i}$, that is to say, $0<V(x(t)) \leqslant 1$; then, according to (9), we obtain

$$
\begin{aligned}
{ }_{0}^{C} D_{t}^{\alpha} V(x(t)) & =\frac{Q_{i} C}{\rho_{i}} D_{t}^{\alpha} x(t)=\frac{Q_{i}}{\rho_{i}}\left[A x(t)+A_{0} x(t-\tau)+B u(t)\right] \\
& =\frac{Q_{i}}{\rho_{i}}\left[(A+B K) x(t)+A_{0} x(t-\tau)\right] \\
& =\frac{1}{\rho_{i}}\left[(F Q)_{i} x(t)+\left(F_{0} Q\right)_{i} x(t-\tau)\right] \\
& =\frac{1}{\rho_{i}}\left(\sum_{j=1}^{n} \sum_{k=1}^{q} F_{i k} Q_{k j} x_{j}(t)+\sum_{j=1}^{n} \sum_{k=1}^{q} F_{0 i k} Q_{k j} x_{j}(t)\right) \\
& =\frac{1}{\rho_{i}}\left(\sum_{k=1}^{q} F_{i k} \sum_{j=1}^{n} Q_{k j} x_{j}(t)+\sum_{k=1}^{q} F_{0 i k} \sum_{j=1}^{n} Q_{k j} x_{j}(t)\right) \\
& \leqslant \frac{1}{\rho_{i}}\left(\sum_{k=1}^{q} F_{i k} \rho_{k}+\sum_{k=1}^{q} F_{0 i k} \rho_{k}\right) \\
& =\frac{1}{\rho_{i}}\left[\left(F+F_{0}\right) \rho\right]_{i} \\
& \leqslant \frac{1}{\rho_{i}}\left(-\varepsilon \rho_{i}\right) \leqslant-\varepsilon V(x(t))<0
\end{aligned}
$$

By Lemma 1, we conclude that the system (8) is asymptotically stable.

Assuming that $x_{0} \in P(Q, \rho)$, i.e., $Q x_{0} \leq \rho$ and $\bar{A}=A+B K$, according to the Definition of $x(t)$, we obtain

$$
\begin{aligned}
Q x(t) & =Q x_{0}+Q\left[\frac{1}{\Gamma(\alpha)} \int_{0}^{t}(t-s)^{\alpha-1}\left[\bar{A} x(s)+A_{0} x(s-\tau)\right] d s\right] \\
& =Q x_{0}+\frac{1}{\Gamma(\alpha)} \int_{0}^{t}(t-s)^{\alpha-1}\left[F Q x(s)+F_{0} Q x(s-\tau)\right] d s \\
& \leqslant \rho+\frac{1}{\Gamma(\alpha)} \int_{0}^{t}(t-s)^{\alpha-1}\left[F Q x(s)+F_{0} Q x(s-\tau)\right] d s \\
& \leqslant \rho+\frac{1}{\Gamma(\alpha)} \int_{0}^{t}(t-s)^{\alpha-1}\left(F \rho+F_{0} \rho\right) d s \\
& \leqslant \rho-\frac{\varepsilon \rho}{\Gamma(\alpha)} \int_{0}^{t}(t-s)^{\alpha-1} d s \\
& =\rho-\frac{\varepsilon \rho t^{\alpha}}{\alpha \Gamma(\alpha)} \leqslant \rho,
\end{aligned}
$$

hence the polyhedron set $P(Q, \rho)$ is a PIS of system (8).

Theorem 3. If there exists a matrix $K \in \mathbb{R}^{m \times n}$, real matrices $F, F_{0} \in \mathbb{R}^{q \times q}$, and a scalar $\varepsilon>0$ such that

$$
\left\{\begin{array}{c}
K(A+B K)=F K \\
K A_{0}=F_{0} K \\
\left(\widehat{F}+\widehat{F_{0}}\right) \widehat{w} \leqslant-\varepsilon \widehat{w}
\end{array}\right.
$$

where $\widehat{w}=\left[\begin{array}{l}w_{2} \\ w_{1}\end{array}\right], \widehat{F}=\left[\begin{array}{ll}F^{+} & F^{-} \\ F^{-} & F^{+}\end{array}\right]$, and

$$
F_{i j}^{+}=\left\{\begin{array}{ll}
F_{i j}, & \text { if } i=j, \\
\max \left(F_{i j}, 0\right), & \text { if } i \neq j,
\end{array} \quad F_{i j}^{-}= \begin{cases}0, & \text { if } i=j, \\
\max \left(-F_{i j}, 0\right), & \text { if } i \neq j,\end{cases}\right.
$$

then the system (8) is asymptotically stable and the polyhedron set $P\left(K,-w_{1}, w_{2}\right)$ is a PIS. 
Proof. From (12), we have

$$
F=F^{+}-F^{-} \text {. }
$$

Replace $F$ in (11) with (13), let $\bar{A}=A+B K$; then, we have

$$
\begin{aligned}
K \bar{A} & =F K=\left(F^{+}-F^{-}\right) K \\
-K \bar{A} & =F(-K)=\left(F^{+}-F^{-}\right)(-K)=\left(F^{-}-F^{+}\right) K .
\end{aligned}
$$

Hence,

$$
\left[\begin{array}{c}
K \\
-K
\end{array}\right] \bar{A}=\left[\begin{array}{ll}
F^{+} & F^{-} \\
F^{-} & F^{+}
\end{array}\right]\left[\begin{array}{c}
K \\
-K
\end{array}\right] .
$$

Let $Q=\left[\begin{array}{c}K \\ -K\end{array}\right], \rho=\widehat{w}=\left[\begin{array}{l}w_{2} \\ w_{1}\end{array}\right]$, that is, the set $P\left(K,-w_{1}, w_{2}\right)$ can be reformulated as the form of $P(Q, \rho)$. From (14), there exists a matrix. $\widehat{F}=\left[\begin{array}{ll}F^{+} & F^{-} \\ F^{-} & F^{+}\end{array}\right]$, such that

$$
Q \bar{A}=\widehat{F} Q
$$

In the same way, we have

$$
Q A_{0}=\widehat{F_{0}} Q \text {. }
$$

On the other hand, according to $\left(\widehat{F}+\widehat{F_{0}}\right) \widehat{w} \leqslant-\varepsilon \widehat{w}$, we obtain

$$
\left(\widehat{F}+\widehat{F_{0}}\right) \rho \leqslant-\varepsilon \rho .
$$

According to (11), we obtain (15)-(17). Then, by Theorem 2, the system (8) is asymptotically stable and the polyhedron set $P(Q, \rho)$ is a PIS. Hence, we conclude that the system (8) is asymptotically stable and the polyhedron set $P\left(K,-w_{1}, w_{2}\right)$ is a PIS.

Theorem 4. The polyhedron sets $P(Q, \rho)$ and $P\left(K,-w_{1}, w_{2}\right)$ have the relation

$$
P(Q, \rho) \subseteq P\left(K,-w_{1}, w_{2}\right)
$$

if and only if there exists $L \in \mathbb{R}^{2 m \times 2 q}, L>0$ such that

$$
\left\{\begin{array}{c}
L\left(\begin{array}{l}
Q \\
Q
\end{array}\right)=\left(\begin{array}{c}
K \\
-K
\end{array}\right), \\
L\left(\begin{array}{l}
\rho \\
\rho
\end{array}\right) \leqslant\left(\begin{array}{l}
w_{2} \\
w_{1}
\end{array}\right) .
\end{array}\right.
$$

Proof. According to Lemma $2, P(Q, \rho) \subseteq P\left(K, w_{2}\right)$ is equivalent to $L_{1} \in \mathbb{R}^{m \times q}, L_{1}>0$ such that $L_{1} Q=K$ and $L_{1} \rho \leqslant w_{2}$. On the other hand, $P(Q, \rho) \subseteq P\left(-K, w_{1}\right)$ is equivalent to $L_{2} \in \mathbb{R}^{m \times q}, L_{2}>0$ such that $L_{2} Q=-K$ and $L_{2} \rho \leqslant w_{1}$.

Hence, $P(Q, \rho) \subseteq P\left(K,-w_{1}, w_{2}\right)$ if and only if there exists $L=\left(\begin{array}{l}L_{1} \\ L_{2}\end{array}\right)>0, L \in R^{2 m \times 2 q}$ such that (18) holds.

Remark 1. Theorem 2 proposes a sufficient condition that ensures the state constraint set $P(Q, \rho)$ is a PIS and the system (8) is asymptotically stable. On this basis, Theorem 3 proposes a sufficient condition that ensures the control constraint set $P\left(K,-w_{1}, w_{2}\right)$ is a PIS and the system (8) is asymptotically stable. Theorem 4 gives a sufficient and necessary condition to ensure $P(Q, \rho) \subseteq P\left(K,-w_{1}, w_{2}\right)$. When considering both state and control constraints. One can use Theorem 2 and Theorem 4 to find a feedback controller for the asymptotic stabilization problem of system (1) according to Corollary 1. When considering only control constraints. One can use Theorem 3 to find a feedback controller for the asymptotic stabilization problem of system (1) according to Corollary 2. 


\section{Design Algorithms}

In this section, two solution algorithms are designed for the state feedback controllers.

Case 1: Considering both the state and control constraints, by Theorem 2, Theorem 4 , and Corollary 1, the solution to matrix inequalities (9) and (18) is the solution to the feedback controller of the asymptotic stabilization problem for system (1). However, for system (1), the rate of convergence to equilibrium is an important index. The largest $\varepsilon$ can ensure the fastest convergence rate to the equilibrium. This can be settled by solving the following LP problem with objective function

$$
S\left(K, F, F_{0}, L, \varepsilon\right)=\varepsilon
$$

and constraints

$$
\left\{\begin{array}{c}
Q(A+B K)=F Q \\
Q A_{0}=F_{0} Q, \\
\left(F+F_{0}\right) \rho \leqslant-\varepsilon \rho, \\
\varepsilon>0 \\
L\left(\begin{array}{c}
Q \\
Q
\end{array}\right)=\left(\begin{array}{c}
K \\
-K
\end{array}\right), \\
L\left(\begin{array}{l}
\rho \\
\rho
\end{array}\right) \leqslant\left(\begin{array}{c}
w_{2} \\
w_{1}
\end{array}\right) .
\end{array}\right.
$$

Case 2: Considering only the control constraints, by Theorem 3 and Corollary 2, the solution to matrix inequality (11) is the solution to the feedback controller of the asymptotic stabilization problem for system (1). This can be found by solving the following NP problem with objective function

$$
S\left(K, F, F_{0}, \varepsilon\right)=\varepsilon
$$

and constraints

$$
\left\{\begin{array}{c}
K(A+B K)=F K, \\
K A_{0}=F_{0} K, \\
\left(\widehat{F}+\widehat{F_{0}}\right) \widehat{w} \leqslant-\varepsilon \widehat{w}, \\
\varepsilon>0 .
\end{array}\right.
$$

Consider the maximum rate of convergence, from the positive definite function

$$
V(x(t))=\max \left\{\max \left(\frac{Q_{1} x(t)}{\rho_{1}}, 0\right), \cdots, \max \left(\frac{Q_{q} x(t)}{\rho_{q}}, 0\right)\right\},
$$

we obtain

$$
\begin{aligned}
{ }_{0}^{C} D_{t}^{\alpha} V(x(t)) & =\frac{Q_{i} C}{\rho_{i}} D_{t}^{\alpha} x(t)=\frac{Q_{i}}{\rho_{i}}\left[A x(t)+A_{0} x(t-\tau)+B u(t)\right] \\
& =\frac{Q_{i}}{\rho_{i}}\left[(A+B K) x(t)+A_{0} x(t-\tau)\right] \\
& =\frac{1}{\rho_{i}}\left[(F Q)_{i} x(t)+\left(F_{0} Q\right)_{i} x(t-\tau)\right] \\
& =\frac{1}{\rho_{i}}\left(\sum_{j=1}^{n} \sum_{k=1}^{q} F_{i k} Q_{k j} x_{j}(t)+\sum_{j=1}^{n} \sum_{k=1}^{q} F_{0 i k} Q_{k j} x_{j}(t)\right) \\
& =\frac{1}{\rho_{i}}\left(\sum_{k=1}^{q} F_{i k} \sum_{j=1}^{n} Q_{k j} x_{j}(t)+\sum_{k=1}^{q} F_{0 i k} \sum_{j=1}^{n} Q_{k j} x_{j}(t)\right) \\
& \leqslant \frac{1}{\rho_{i}}\left(\sum_{k=1}^{q} F_{i k} \rho_{k}+\sum_{k=1}^{q} F_{0 i k} \rho_{k}\right) \\
& =\frac{1}{\rho_{i}}\left[\left(F+F_{0}\right) \rho\right]_{i} \\
& \leqslant \frac{1}{\rho_{i}}\left(-\varepsilon \rho_{i}\right) \leqslant-\varepsilon V(x(t))<0
\end{aligned}
$$

Therefore, maximizing $\varepsilon$ is to maximize the rate of convergence. 
Remark 2. When the system parameters $A, A_{0}$, and $B$ and the constraint parameters $Q, \rho$, and $\widehat{w}$ are fixed, from (23) we can see that the parameter $\varepsilon$ is closely related to the rate of convergence. If $0<\varepsilon<1$, the largest $\varepsilon$ can ensure the fastest convergence rate to the equilibrium.

\section{Numerical Examples}

Example 1. Consider the delayed fractional-order electrical circuit [36] shown in Figure 1 with the given resistance $R=1 \Omega$, inductance $L=0.4167 \mathrm{mH}$, capacitance $C=1.67 \mathrm{mF}$, delay element and source voltage $u$.

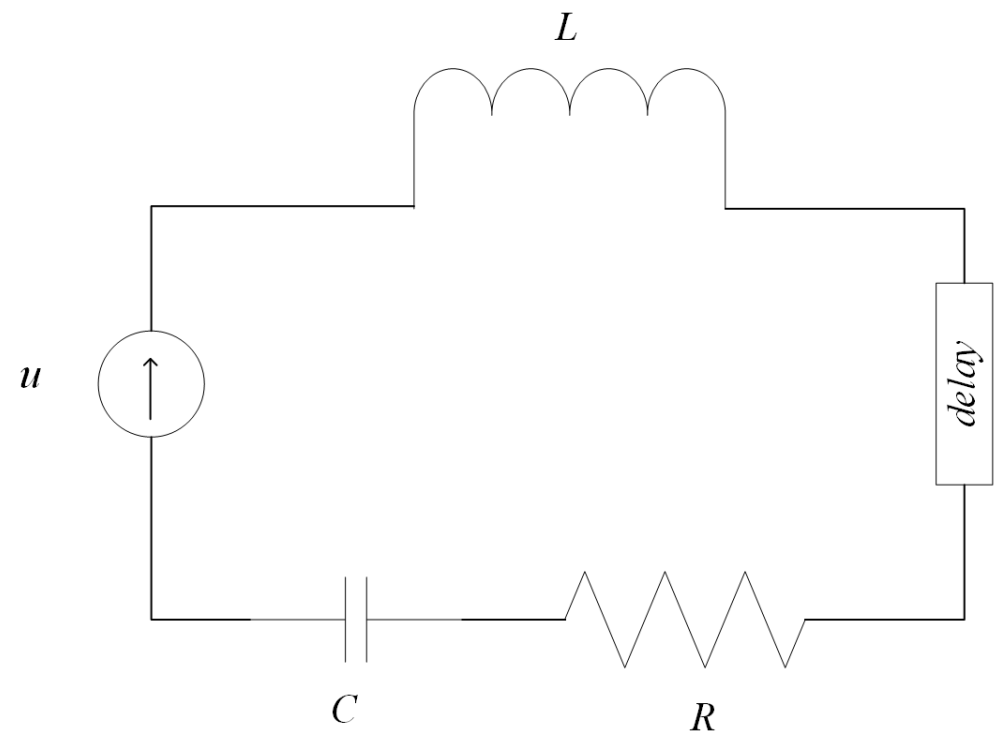

Figure 1. The delayed fractional-order electrical circuit in [36].

Let $v(t)$ represent the voltage across the capacitor and $i(t)$ represent the current passing through the inductor, using Kirchhoff's laws, we can write the circuit equations

$$
\left[\begin{array}{c}
\frac{d^{\alpha} v(t)}{d t^{\alpha}} \\
\frac{d^{\alpha} i(t)}{d t^{\alpha}}
\end{array}\right]=A\left[\begin{array}{l}
v(t) \\
i(t)
\end{array}\right]+A_{0}\left[\begin{array}{c}
v(t-\tau) \\
i(t-\tau)
\end{array}\right]+B u(t)
$$

where $A=\left[\begin{array}{cc}0 & \frac{1}{C} \\ -\frac{1}{L} & -\frac{R}{L}\end{array}\right], A_{0}=\left[\begin{array}{cc}0 & -\frac{1}{R} \\ -\frac{C}{L} & 0\end{array}\right], B=\left[\begin{array}{l}0 \\ \frac{1}{L}\end{array}\right]$.

Let $x_{1}(t)=v(t), x_{2}(t)=i(t)$. Then, the above circuit equations can be written in the form of the DLFS (1), with $\alpha=0.7, \tau=0.1, A=\left[\begin{array}{cc}0 & 0.6 \\ -2.4 & -2.4\end{array}\right], A_{0}=\left[\begin{array}{cc}0 & -1 \\ -4 & 0\end{array}\right]$, and $B=\left[\begin{array}{c}0 \\ 2.4\end{array}\right]$.

Suppose the control input $P(K,-\underline{w}, \bar{w})$ satisfies the constraint

$$
-100 \leqslant K x(t) \leqslant 50 .
$$

Without the control input, system (1) is unstable; for the initial condition $(4,1)^{T}$, the time response of the state is shown in Figure 2.

The asymptotic stabilization problem of DLFS (1) is to find a controller $u(t)=K x(t)$ that makes all trajectories starting with the initial condition $x_{0}$ asymptotically stable and meanwhile satisfy the control constraints (24).

According to Case 2, solving the NP problem with the objective function

$$
S\left(K, F, F_{0}, \varepsilon\right)=\varepsilon
$$


and under the constraints

$$
\left\{\begin{array}{c}
K\left(\left[\begin{array}{cc}
0 & 0.6 \\
-2.4 & -2.4
\end{array}\right]+\left[\begin{array}{c}
0 \\
2.4
\end{array}\right] K\right)=F K \\
K\left[\begin{array}{cc}
0 & -1 \\
-4 & 0
\end{array}\right]=F_{0} K \\
\left(\left[\begin{array}{cc}
F^{+} & F^{-} \\
F^{-} & F^{+}
\end{array}\right]+\left[\begin{array}{cc}
F_{0}^{+} & F_{0}^{-} \\
F_{0}^{-} & F_{0}^{+}
\end{array}\right]\right)\left[\begin{array}{c}
50 \\
100
\end{array}\right] \leqslant-\varepsilon\left[\begin{array}{c}
50 \\
100
\end{array}\right] \\
\varepsilon>0 .
\end{array}\right.
$$

By calculation, we obtain $\varepsilon=0.8, K=\left[\begin{array}{ll}2 & 1\end{array}\right], F=1.2$, and $F_{0}=-2$.

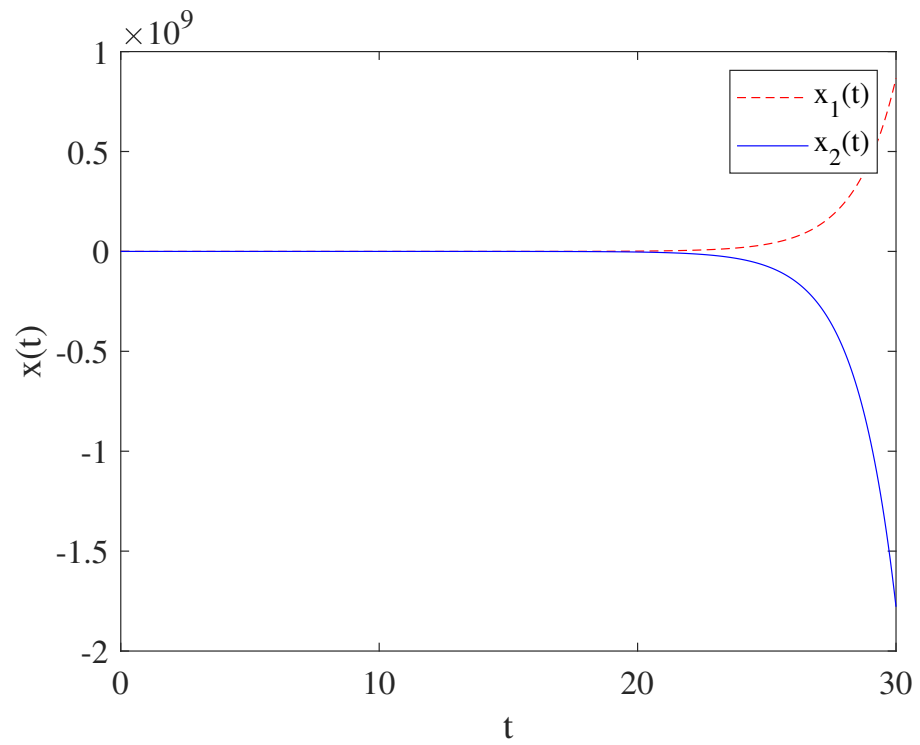

Figure 2. The time response of the system state without feedback control.

It indicates that there exists a $u(t)=\left[\begin{array}{ll}2 & 1\end{array}\right] x(t)$ such that all trajectories originating from the initial condition are asymptotically stable to the origin, and meanwhile the corresponding trajectory satisfies the control constraints (24).

For the initial condition $(4,1)^{T}$, the time response of the state is shown in Figure 3.

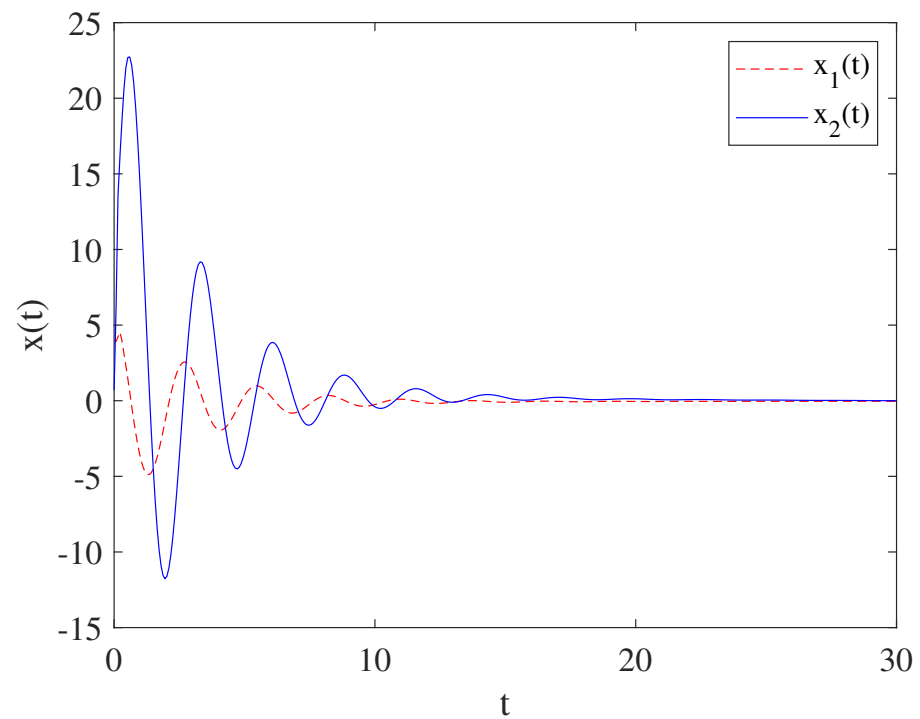

Figure 3. The time response of the system state with feedback control.

Additionally, $u(t)$ satisfies the control constraints (24), which can be seen from Figure 4. 


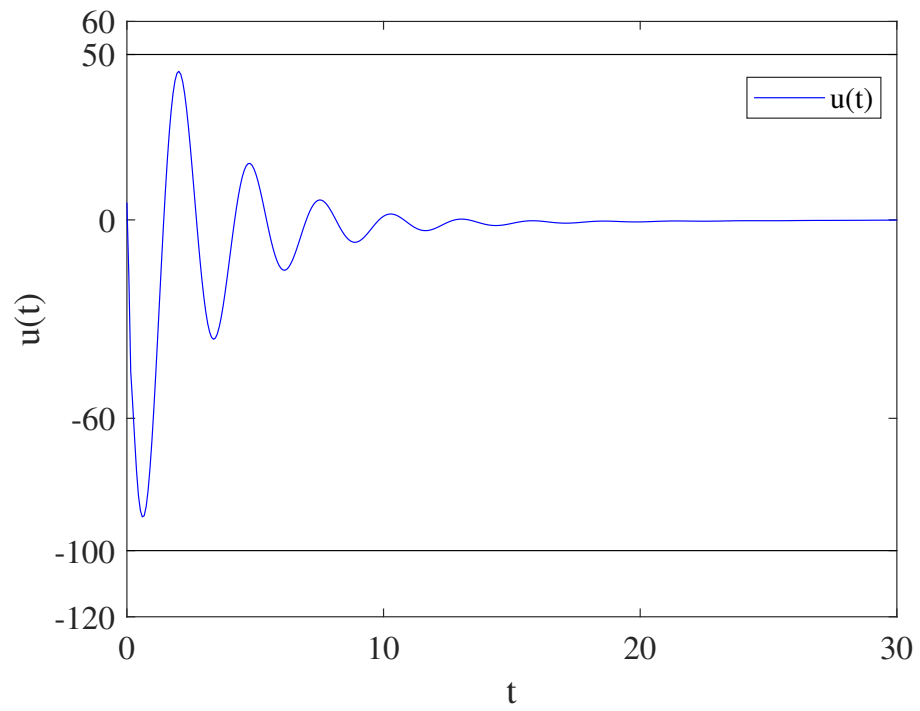

Figure 4. The control input $u(t)$.

So, $u(t)=\left[\begin{array}{ll}2 & 1\end{array}\right] x(t)$ is a solution to the asymptotic stabilization problem of DLFS (1).

Example 2. Consider another delayed fractional-order electrical circuits, which can be written in the form of the DLFS (1), with $\alpha=0.7, \tau=0.5, A=\left[\begin{array}{cc}-3.25 & 10 \\ -1 & 7.1\end{array}\right], A_{0}=\left[\begin{array}{cc}-1 & -2.2 \\ 0 & -2.1\end{array}\right]$, and $B=\left[\begin{array}{c}1 \\ 0.8\end{array}\right]$.

The state constraints $P(Q, \rho)$ are

$$
\begin{aligned}
2 x_{2}(t) & \leqslant 0.8, \\
-x_{1}(t)+2 x_{2}(t) & \leqslant 2.4, \\
-0.5 x_{1}(t)+4 x_{2}(t) & \geqslant-1.8,
\end{aligned}
$$

where $Q=\left[\begin{array}{cc}0 & 2 \\ -1 & 2 \\ 0.5 & -4\end{array}\right] \in R^{3 \times 2}$ and $\rho=\left[\begin{array}{l}0.8 \\ 2.4 \\ 1.8\end{array}\right] \in R^{3}, \rho>0$. It can be easily seen that the polyhedron set $P(Q, \rho)$ is closed and nonempty.

Suppose the control input $P(K,-\underline{w}, \bar{w})$ satisfies the constraint

$$
-6 \leqslant K x(t) \leqslant 9
$$

Without the control input, system (1) is unstable, the time response of the state with the initial condition $(6.8,0.4)^{T}$ is shown in Figure 5.

The asymptotic stabilization problem of DLFS (1) is to find a controller $u(t)=K x(t)$ that makes all trajectories starting from the initial value $x_{0}$ asymptotically stable and meanwhile satisfy the state constraints (25) and the control constraints (26).

According to Case 1, solving the LP problem with the objective function

$$
S\left(K, F, F_{0}, L, \varepsilon\right)=\varepsilon
$$

and under the constraints 


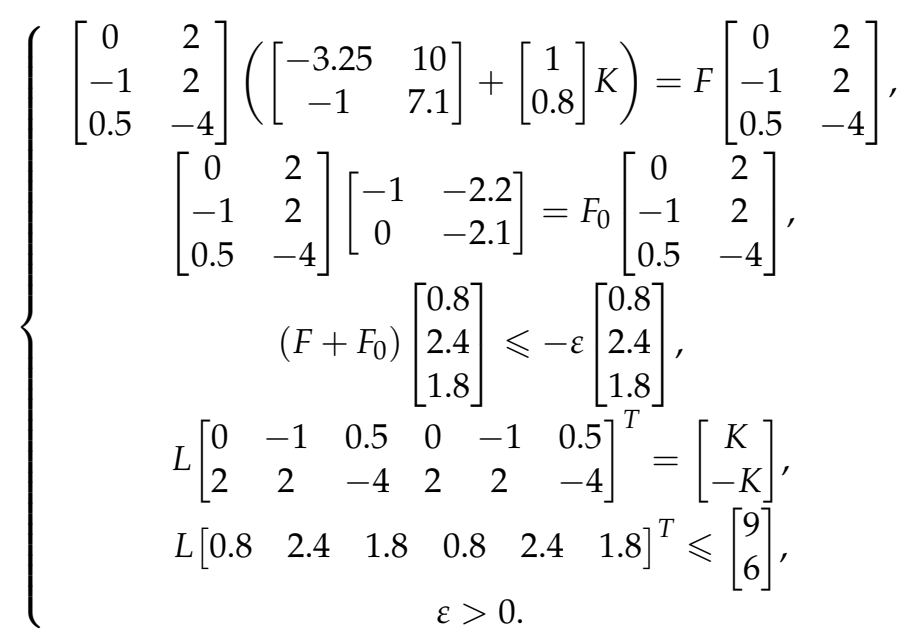

By computation, we obtain

$$
\begin{gathered}
\varepsilon=0.9, \\
K=\left[\begin{array}{ll}
1.25 & -10
\end{array}\right], \\
F=\left[\begin{array}{ccc}
-0.9 & 0 & 0 \\
1.1 & -2 & 0 \\
0 & 0.7 \dot{3} & -0.5 \dot{3}
\end{array}\right], \\
F_{0}=\left[\begin{array}{ccc}
-2.1 & 0 & 0 \\
0 & -1 & 0 \\
0 & -0.55 & -2.1
\end{array}\right],
\end{gathered}
$$

and

$$
L=\left[\begin{array}{cccccc}
0 & 0 & 0 & 0 & 0 & 2.5 \\
0 & 0 & 0 & 3.75 & 1.25 & 0
\end{array}\right]
$$

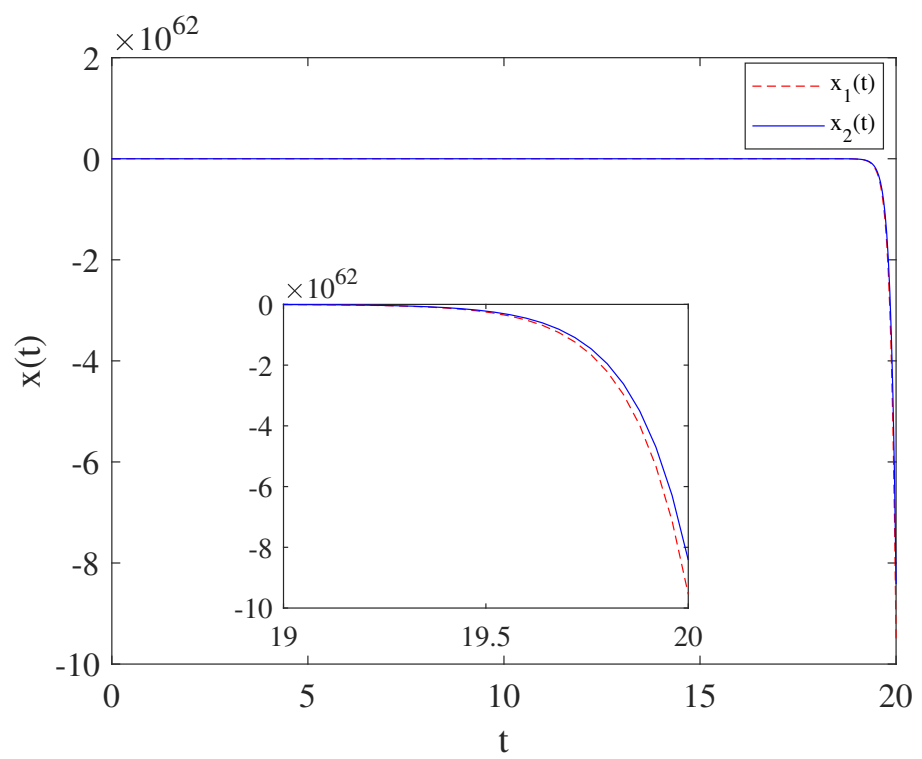

Figure 5. The time response of the system state without feedback control.

It suggests that there exists $u(t)=1.25 x_{1}(t)-10 x_{2}(t)$ such that all trajectories starting from the initial condition are asymptotically stable to the origin, and meanwhile the corresponding state trajectory satisfies the state constraints (25) and the control constraints (26).

For the initial condition $(6.8,0.4)^{T}$, the time response of the state is shown in Figure 6. 


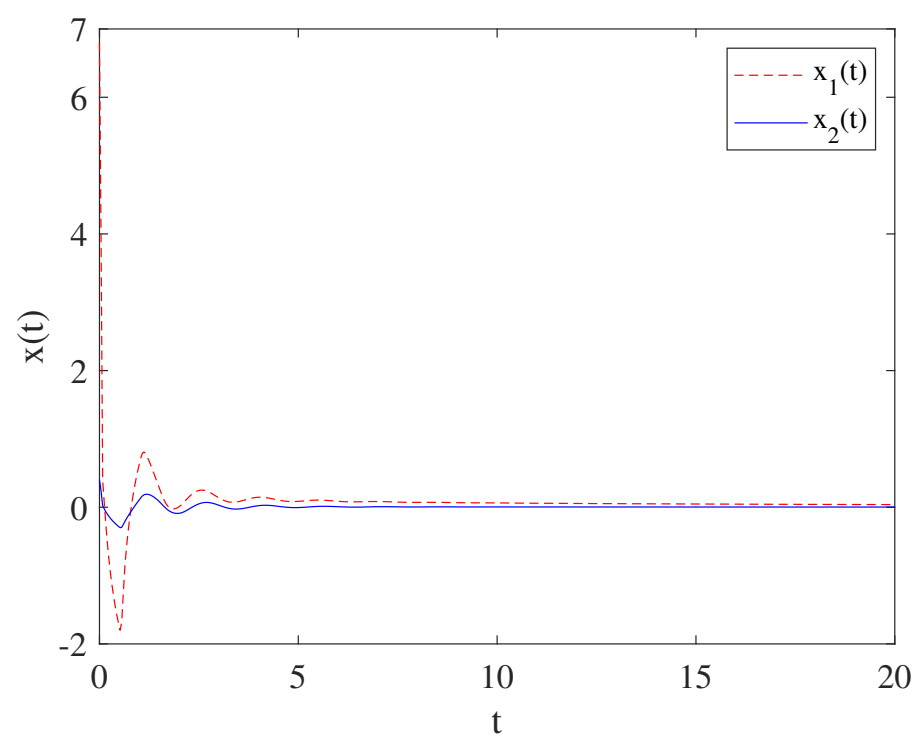

Figure 6. The time response of the system state with feedback control.

For the initial condition $(6.8,0.4)^{T}$, the corresponding phase trajectory which satisfies the state constraints (25) and the control constraints (26), is shown in Figure 7.

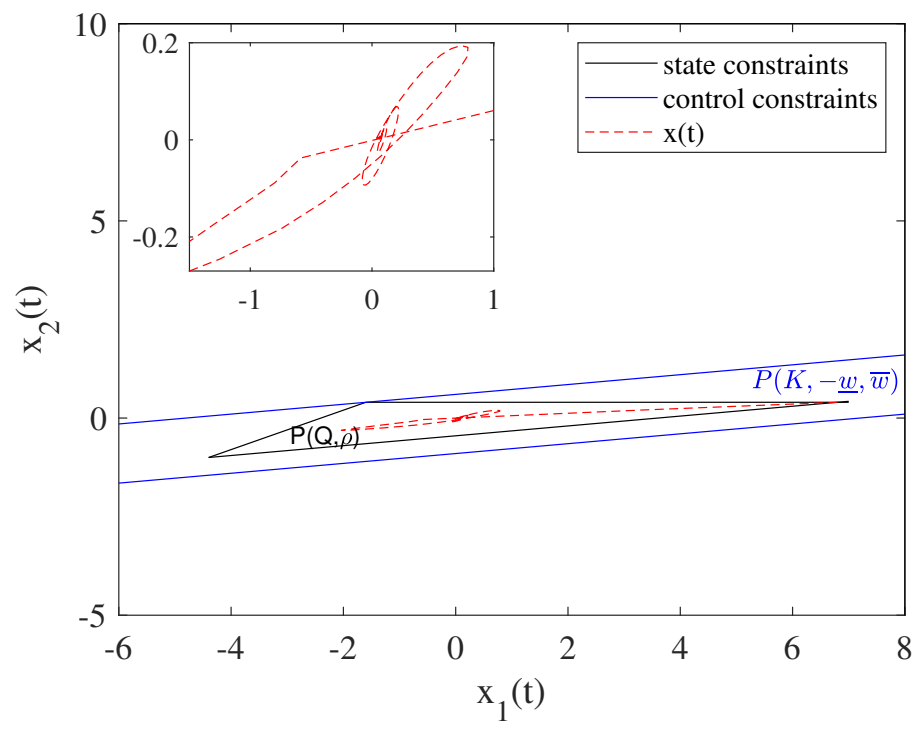

Figure 7. The phase trajectory with the initial condition $(6.8,0.4)^{T}$.

Furthermore, $u(t)$ satisfies the control constraints (26), which can be seen in Figure 8.

Hence, $u(t)=1.25 x_{1}(t)-10 x_{2}(t)$ is a solution to the asymptotic stabilization problem of DLFS (1).

Clearly, from Example 2, it can be seen that the control input is not saturated because the state constraint set is a PIS. Next, we give an example without state constraints to verify the saturation of the input control. 


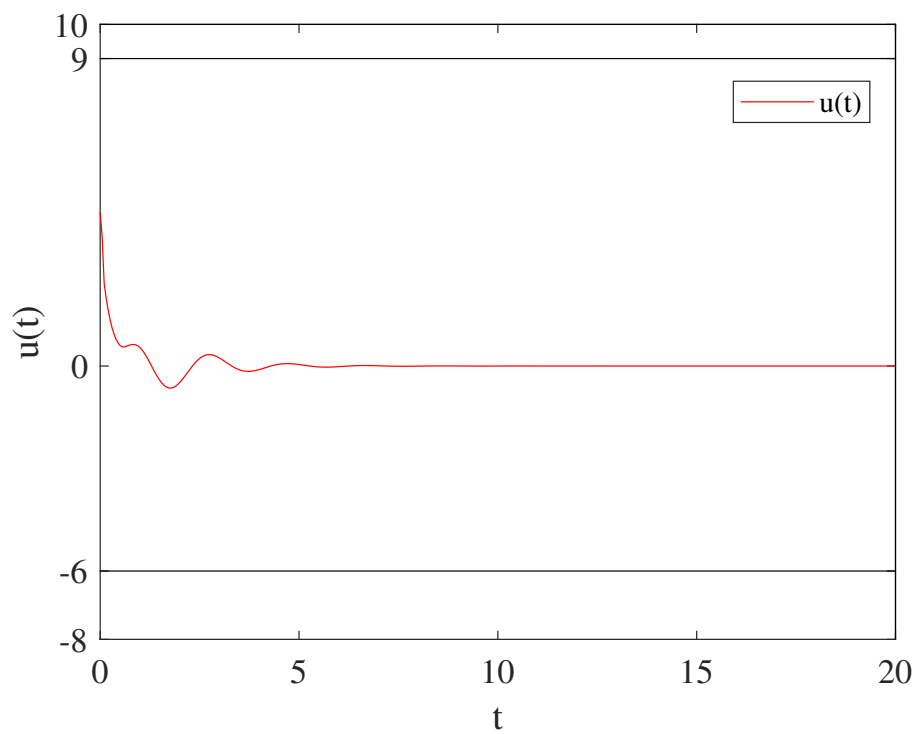

Figure 8. The control input $u(t)$.

Example 3. Consider the DLFS (1), with $\alpha=0.8, \tau=0.8, A=\left[\begin{array}{cc}1 & -3 \\ -1 & 5\end{array}\right], A_{0}=\left[\begin{array}{cc}-1 & 0 \\ 0 & -2\end{array}\right]$, and $B=\left[\begin{array}{cc}1 & 1 \\ -1 & 0.5\end{array}\right]$.

Suppose the control input $P\left(K,-w_{1}, w_{2}\right)$ satisfies the constraint

$$
\left[\begin{array}{l}
-1 \\
-2
\end{array}\right] \leqslant K x(t) \leqslant\left[\begin{array}{l}
2 \\
4
\end{array}\right]
$$

Without the control input, system (1) is unstable; for the initial condition $(-1.84,-0.64)^{T}$, the time response of the state is shown in Figure 9.

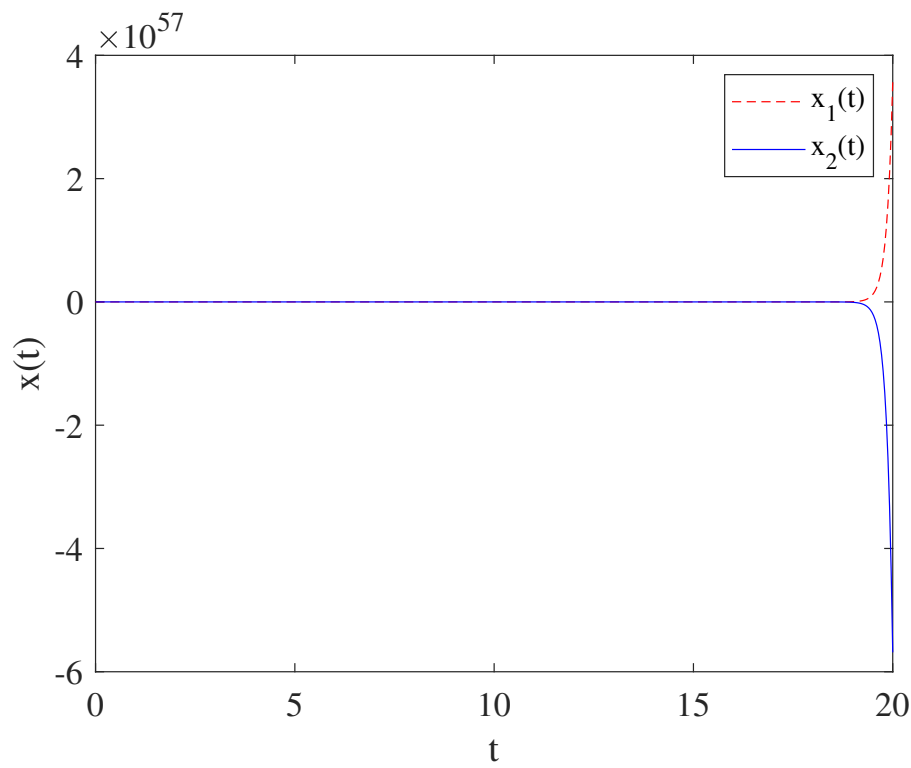

Figure 9. The time response of the system state without feedback control.

The asymptotic stabilization problem of DLFS (1) is to find a controller $u(t)=K x(t)$ that makes all trajectories starting with the initial condition $x_{0}$ asymptotically stable and meanwhile satisfy the control constraints (27). 
According to Case 2, solving the NP problem with the objective function

$$
S\left(K, F, F_{0}, \varepsilon\right)=\varepsilon
$$

and under the constraints

$$
\left\{\begin{array}{c}
K\left(\left[\begin{array}{cc}
1 & -3 \\
-1 & 5
\end{array}\right]+\left[\begin{array}{cc}
1 & 1 \\
-1 & 0.5
\end{array}\right] K\right)=F K \\
K\left[\begin{array}{cc}
-1 & 0 \\
0 & -2
\end{array}\right]=F_{0} K \\
\left(\left[\begin{array}{ll}
F^{+} & F^{-} \\
F^{-} & F^{+}
\end{array}\right]+\left[\begin{array}{ll}
F_{0}^{+} & F_{0}^{-} \\
F_{0}^{-} & F_{0}^{+}
\end{array}\right]\right)\left[\begin{array}{l}
2 \\
4 \\
1 \\
2
\end{array}\right] \leqslant-\varepsilon\left[\begin{array}{l}
2 \\
4 \\
1 \\
2
\end{array}\right] \\
\varepsilon>0 .
\end{array}\right.
$$

By calculation, we obtain

$$
\begin{gathered}
\varepsilon=0.8908 \\
K=\left[\begin{array}{cc}
-2.051 & 7.444 \\
-1.255 & -2.629
\end{array}\right], \\
F=\left[\begin{array}{cc}
-4.11 & 0.43 \\
0.33 & -1.96
\end{array}\right]
\end{gathered}
$$

and

$$
F_{0}=\left[\begin{array}{cc}
-1.634 & 1.0362 \\
0.2239 & -1.3660
\end{array}\right]
$$

It indicates that there exists a $u(t)=\left[\begin{array}{cc}-2.051 & 7.444 \\ -1.255 & -2.629\end{array}\right] x(t)$ such that all trajectories originating from the initial condition are asymptotically stable to the origin, and meanwhile the corresponding trajectory satisfies the control constraints (27).

For the initial condition $(-1.84,-0.64)^{T}$, the time response of the state is shown in Figure 10.

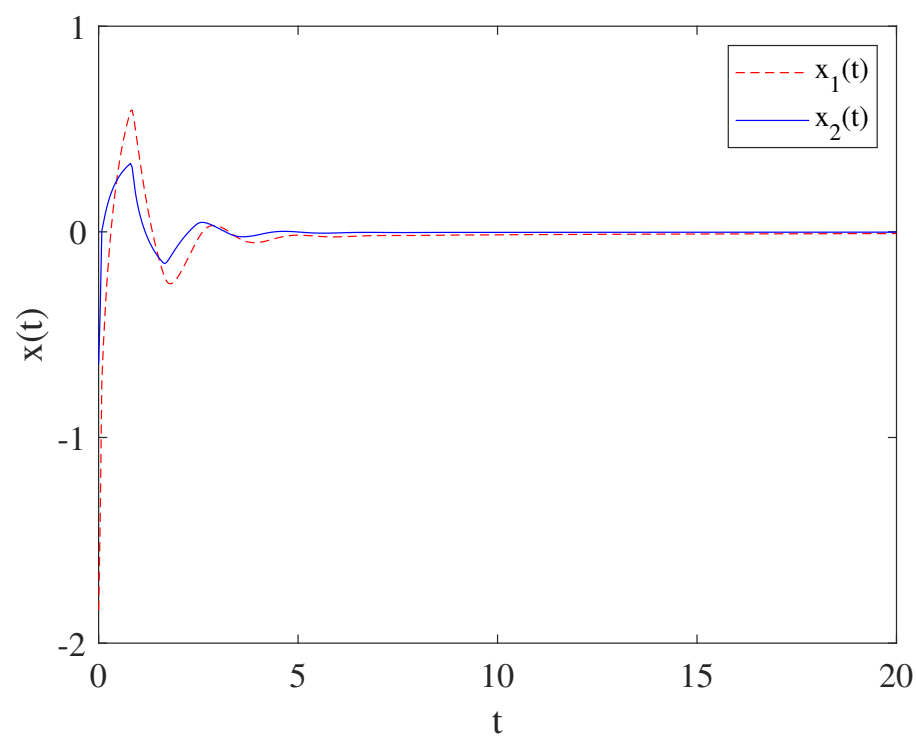

Figure 10. The time response of the system state with feedback control.

For the initial condition $(-1.84,-0.64)^{T}$, the corresponding phase trajectory is shown in Figure 11. It can be seen that the control constraints (27) is satisfied. 


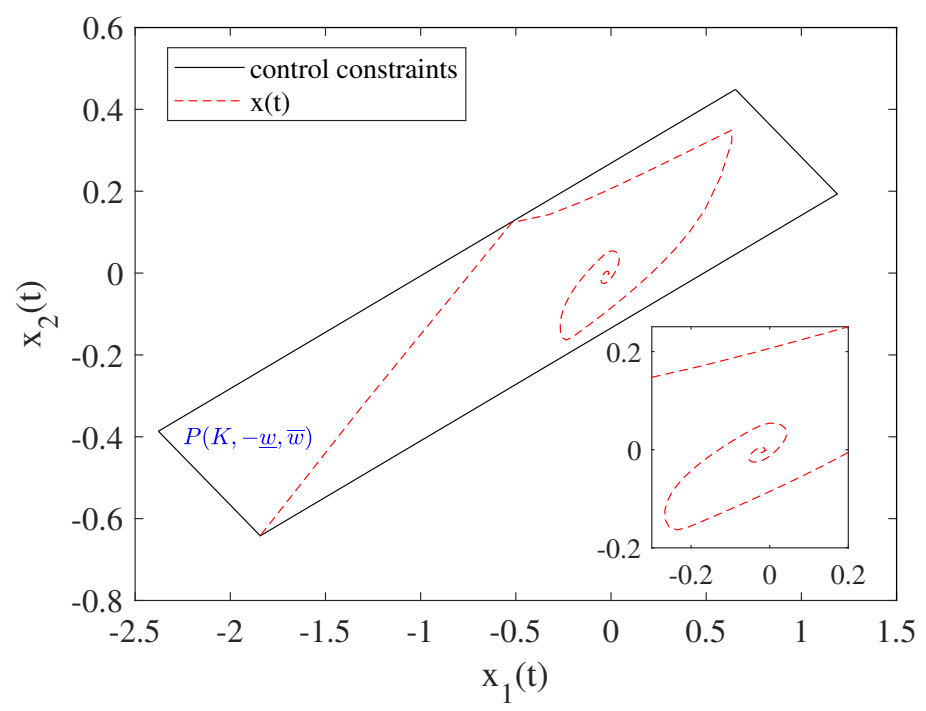

Figure 11. The phase trajectory with the initial condition $(-1.84,-0.64)^{T}$.

In addition, $u(t)$ satisfies the control constraints (27), which can be seen from Figure 12.
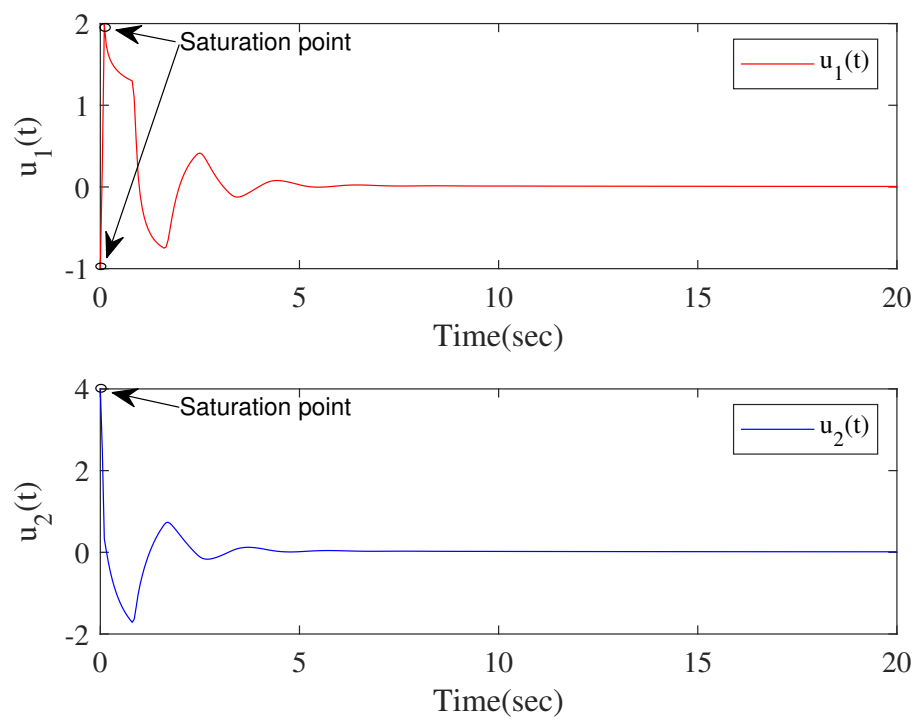

Figure 12. The control input $u(t)$. DLFS (1).

So, $u(t)=\left[\begin{array}{cc}-2.051 & 7.444 \\ -1.255 & -2.629\end{array}\right] x(t)$ is a solution to the asymptotic stabilization problem of

\section{Conclusions}

The asymptotic stabilization problem of DLFS subject to state and control constraints is studied. Based on the invariant set theory and fractional-order Lyapunov stability theory, the feedback controller and the corresponding solution algorithms that ensure the asymptotic stability of the DLFS under constraints are given. Numerical examples show that the proposed method is effective. Stability of delayed fractional-order neural network systems with constraints are a very interesting topic. In the future, we will study the stabilization of delayed fractional-order neural network systems subject to constraints by using the invariant set method. 
Author Contributions: Conceptualization, X.S. and Z.W.; methodology, X.S. and Z.W.; software, X.S.; validation, X.S., Z.W., Z.S. and Z.Z.; investigation, Z.W.; writing-original draft preparation, X.S.; writing-review and editing, Z.W., Z.S. and Z.Z.; supervision, Z.W.; funding acquisition, Z.W. All authors have read and agreed to the published version of the manuscript.

Funding: This work was supported by the National Natural Science Foundation of China under Grants 61973199, 62003794, 62173214, the Shandong Provincial Natural Science Foundation ZR2020QF050, ZR2021MF003 and the Taishan Scholar Project of Shandong Province of China.

Institutional Review Board Statement: Not applicable.

Informed Consent Statement: Not applicable.

Data Availability Statement: Data sharing not applicable to this article as no datasets were generated or analysed during the current study.

Acknowledgments: We would like to express our great appreciation to the editors and reviewers.

Conflicts of Interest: The authors declare no conflicts of interest.

Abbreviations
The following abbreviations are used in this man
DLFS delayed linear fractional-order systems
PIS positive invariant sets
LP linear programming
NP nonlinear programming
LMI linear matrix inequality

\section{References}

1. Chen, L.; Chen, Y.; Lopes, A.M.; Kong, H.; Wu, R. State of charge estimation of lithium-ion batteries based on fuzzy fractional-order unscented kalman filter. Fractal Fract. 2021, 5, 91.

2. Tian, Y.; Xia, Q.; Chai, Y.; Chen, L.; Lopes, A.M.; Chen, Y. Guaranteed cost leaderless consensus protocol design for fractional-order uncertain multi-agent systems with state and input delays. Fractal Fract. 2021, 5, 141.

3. Gonzalez-Acuna, R.G.; Davila, A.; Gutierrez-Vega, J.C. Optical flow of non-integer order in particle image velocimetry techniques. Signal Process. 2019, 155, 317-322. [CrossRef]

4. Lashkarian, E.; Hejazi, S.R.; Habibi, N.; Motamednezhad, A. Symmetry properties, conservation laws, reduction and numerical approximations of time-fractional cylindrical-burgers equation. Commun. Nonlinear Sci. Numer. Simul. 2019, 67, 176-191. [CrossRef]

5. Yin, C.; Dadras, S.; Cheng, Y.H.; Huang, X.G.; Cao, J.W.; Malek, H. Multidimensional fractional-order newton-based extremum seeking for online light-energy saving technique of lighting system. IEEE Trans. Ind. Electron. 2019, 67, 8576-8586. [CrossRef]

6. Mohsenipour, R.; Jegarkandi, M.F. Robust stability analysis of fractional-order interval systems with multiple time delays. Int. J. Robust Nonlinear Control 2019, 29, 1823-1839. [CrossRef]

7. Sakthivel, R.; Mohanapriya, S.; Ahn, C.K.; Karimi, H.R. Output tracking control for fractional-order positive switched systems with input time delay. IEEE Trans. Circuits Syst. II-Express Briefs 2019, 66, 1013-1017. [CrossRef]

8. Jia, J.; Huang, X.; Li, Y.; Cao, J. Global stabilization of fractional-order memristor-based neural networks with time delay. IEEE Trans. Neural Netw. Learn. Syst. 2020, 31, 997-1009. [CrossRef]

9. Wang, Z.; Wang, X.; Xia, J.; Shen, H.; Meng, B. Adaptive sliding mode output tracking control based-FODOB for a class of uncertain fractional-order nonlinear time-delayed systems. Sci. China-Technol. Sci. 2020, 63, 1854-1862. [CrossRef]

10. Liu, H.; Pan, Y.; Cao, J.; Zhou, Y.; Wang, H. Positivity and stability analysis for fractional-order delayed systems: A T-S fuzzy model approach. IEEE Trans. Fuzzy Syst. 2020, 29, 927-939. [CrossRef]

11. Du, F.; Lu, J. Finite-time stability of neutral fractional order time delay systems with Lipschitz nonlinearities. Appl. Math. Comput. 2020, 375, 125079. [CrossRef]

12. Naifar, O.; Nagy, A.M.; Makhlouf, A.B.; Kharrat, M. Finite-time stability of linear fractional-order time-delay systems. Int. J. Robust Nonlinear Control 2018, 29, 180-187. [CrossRef]

13. Liu, P.; Zeng, Z.; Wang, J. Asymptotic and finite-time cluster synchronization of coupled fractional-order neural networks with time delay. IEEE Trans. Neural Netw. Learn. Syst. 2020, 31, 4956-4967. [CrossRef]

14. Thanh, N.T.; Phat, V.N.; Niamsup, P. New finite-time stability analysis of singular fractional differential equations with timevarying delay. Fract. Calc. Appl. Anal. 2020, 23, 504-519. [CrossRef]

15. Deng, W.; Li, C.; Lu, J. Stability analysis of linear fractional differential system with multiple time delays. Nonlinear Dyn. 2007, 48, 409-416. [CrossRef] [PubMed] 
16. You, X.; Song, Q.; Zhao, Z. Global Mittag-Leffler stability and synchronization of discrete-time fractional-order complex-valued neural networks with time delays. Neural Netw. 2020, 122, 382-394. [CrossRef]

17. Hu, T.; He, Z.; Zhang, X.; Zhong, S. Finite-time stability for fractional-order complex-valued neural networks with time delay. Appl. Math. Comput. 2020, 365, 124715. [CrossRef]

18. Bonnet, C.; Partington, J.R. Stabilization of some fractional delay systems of neutral type. Automatica 2007, 43, 2047-2053. [CrossRef] [PubMed]

19. He, B.; Zhou, H.; Kou, C.; Chen, Y.Q. New integral inequalities and asymptotic stability of fractional-order systems with unbounded time delay. Nonlinear Dyn. 2018, 94, 1523-1534. [CrossRef]

20. Chen, L.; Wu, R.; Cheng, Y.; Chen, Y.Q. Delay-dependent and order-dependent stability and stabilization of fractional-order linear systems with time-varying delay. IEEE Trans. Circuits Syst. II-Express Briefs 2020, 67, 1064-1068. [CrossRef]

21. Tavazoei, M.S.; Badri, V. Stability analysis of fractional order time-delay systems: Constructing new lyapunov functions from those of integer order counterparts. IET Control Theory Appl. 2019, 13, 2476-2481. [CrossRef]

22. Nie, X.; Liu, P.; Liang, J.; Cao, J. Exact coexistence and locally asymptotic stability of multiple equilibria for fractional-order delayed Hopfield neural networks with Gaussian activation function. Neural Netw. 2021, 142, 690-700. [CrossRef]

23. Hennet, J.C.; Beziat, J.P. A class of invariant regulators for the discrete-time linear constrained regulation problem. Automatica 1991, 27, 549-554.

24. Vassilaki, M.; Bitsoris, G. Constrained regulation of linear continuous-time dynamical systems. Syst. Control Lett. 1989, 13, 247-252. [CrossRef] [PubMed]

25. Bitsoris, G.; Olaru, S. Further results on the linear constrained regulation problem. In Proceedings of the 21st Mediterranean Conference on Control and Automation, Crete, Greece, 25-28 June 2013; pp. 824-830. [CrossRef]

26. Bitsoris, G.; Olaru, S.; Vassilaki, M. On the linear constrained regulation problem for continuous-time systems. In Proceedings of the 19th World Congress International Federation of Automatic Control, Cape Town, South Africa, 24-29 August 2014; pp. 4004-4009. [CrossRef]

27. Castelan, E.B. On invariant polyhedra of continuous-time linear systems. IEEE Trans. Autom. Control 1991, 38, $1680-1685$.

28. Blanchini, F. Set invariance in control. Automatica 1999, 35, 1747-1767.

29. Jia, J.; Wang, F.; Zeng, Z. Global stabilization of fractional-order memristor-based neural networks with incommensurate orders and multiple time-varying delays: A positive-system-based approach. Nonlinear Dyn. 2021, 104, 2303-2329. [CrossRef]

30. Zhang, X.; Zhao, Z. Normalization and stabilization for rectangular singular fractional order T-S fuzzy systems. Fuzzy Sets Syst. 2019, 381, 140-153. [CrossRef]

31. Zhang, Z.; Zhang, J. Asymptotic stabilization of general nonlinear fractional-order systems with multiple time delays. Nonlinear Dyn. 2020, 102, 605-619. [CrossRef]

32. Lim, Y.H.; Ahn, H.S. On the positive invariance of polyhedral sets in fractional-order linear systems. Automatica 2013, 49, 3690-3694. [CrossRef]

33. Yan, L.; Chen, Y.Q.; Podlubny, I. Stability of fractional-order nonlinear dynamic systems: Lyapunov direct method and generalized Mittag-Leffler stability. Comput. Math. Appl. 2010, 59, 1810-1821.

34. Wen, Y.; Zhou, X.F.; Zhang, Z.; Liu, S. Lyapunov method for nonlinear fractional differential systems with delay. Nonlinear Dyn. 2015, 82, 1015-1025. [CrossRef]

35. Athanasopoulos, N.; Bitsoris, G.; Vassilaki, M. Stabilization of bilinear continuous-time systems. In Proceedings of the 18th Mediterranean Conference on Control and Automation, MED'10, Marrakech, Morocco, 23-25 June 2010; pp. $442-447$.

36. Kaczorek, T.; Rogowski, K. Fractional Linear Systems and Electrical Circuits; Springer: New York, NY, USA, $2014 ;$ p. 65. 